\title{
Three-Dimensional Digital Subtraction Angiography for Endovascular Treatment of Direct Carotid-Cavernous Fistula -Case Report-
}

\author{
Shinya KOHYAMA, Shoichiro IsHIHARA, Fumitaka YAMANE, \\ Ryuzaburo KANAZAWA, Hideaki ISHIHARA, and Masanori SUZUKI \\ Division of Endovascular Neurosurgery, Stroke Center, International Medical Center, \\ Saitama Medical University, Hidaka, Saitama
}

\begin{abstract}
A 63-year-old woman with a direct carotid-cavernous fistula (CCF), manifesting as sudden onset of severe headache and pulsatile tinnitus suffered during coronary angiography, was successfully treated with transarterial embolization based on the anatomical information obtained from volume-rendered three-dimensional digital subtraction angiography (3D DSA) images. 3D DSA is useful for understanding the configuration of such fistulas accurately and devising a therapeutic strategy for the endovascular treatment of direct CCFs.
\end{abstract}

Key words: carotid-cavernous fistula, three-dimensional digital subtraction angiography, coil embolization, transarterial approach 


\section{Introduction}

Direct carotid-cavernous fistula (CCF) is relatively rare, but may show progressive severe symptoms such as visual impairment, ophthalmoplegia, and cerebral ischemia or hemorrhage due to venous hypertension. ${ }^{6)}$ Most direct CCFs are traumatic in origin, and are less commonly caused by rupture of an aneurysm of the cavernous segment. ${ }^{2)}$ Endovascular transarterial occlusion is now the first-choice treatment for direct CCFs. ${ }^{2}$ The detachable balloon has been used to obliterate fistulas, but the platinum coil is considered a promising alternative because of its better controllability. ${ }^{8)}$ Coil embolization, a well-established therapeutic option for cerebral aneurysms, is considered feasible, especially in cases of direct CCFs caused by a ruptured aneurysm. Understanding of the configuration of the fistula is important to avoid inadequate packing in the cavernous sinus, which may cause worsening of symptoms due to compression of the cranial nerves or incomplete occlusion of the fistula. ${ }^{4)}$

This study describes the utility of three-dimensional digital subtraction angiography (3D DSA) for providing anatomical information of direct CCF for transarterial coil embolization.

\section{Case Report}

A 63-year-old woman with a medical history of percutaneous coronary intervention for myocardial infarction suffered sudden onset of severe headache and pulsatile tinnitus during coronary angiography. Further neuroradiological examination was not performed because computed tomography (CT) of the brain showed no abnormalities, and the headache subsided in a few days without other new symptoms. However, the pulsatile tinnitus continued, and she developed visual impairment, chemosis, and exophthalmus on the right after 4 months. She consulted an ophthalmologist because these symptoms gradually worsened within a month. CT with contrast medium showed an enlarged right superior ophthalmic vein (SOV), so she was referred to our institute.

Conventional DSA demonstrated a direct CCF on the right, draining anteriorly to the ipsilateral SOV, and posteriorly to the inferior petrosal sinus (Fig. 1A). Direct CCF was diagnosed originating from the ruptured aneurysm on the cavernous segment of the internal carotid artery (ICA), based on the clinical course with sudden onset of symptoms without head injury. Symptoms were thought to have progressed gradually, according to the obstruction of the draining venous routes.

Transarterial coil embolization was attempted under general anesthesia. 3D DSA was performed using an Axion Artis dBA detector (Siemens AG, Munich, Germany) to detect the fistulous point (Fig. 1B), using a 5-second $190^{\circ}$ rotational run, acquisition of 126 images, and injection of $3.5-\mathrm{ml}$ contrast material per second in the ICA. 3D reconstructions were performed in a $512 \times 512$ matrix, on a dedicated workstation.

Adjustment of the window level of 3D DSA showed the fistulous point on the lateral wall of the right ICA at the
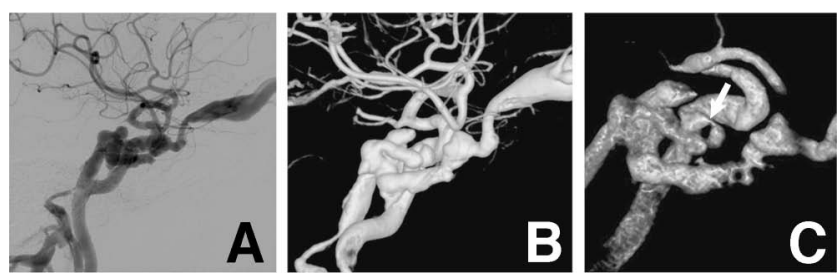

Fig. 1 Lateral views of conventional digital subtraction angiography (DSA) (A) and three-dimensional (3D) DSA (B) of the right internal carotid artery showing a cavernous sinus dural arteriovenous fistula draining into the superior ophthalmic vein and the inferior petrosal sinus, and 3D DSA (C) showing the fistula (arrow) detected by controlling the angle and window level.

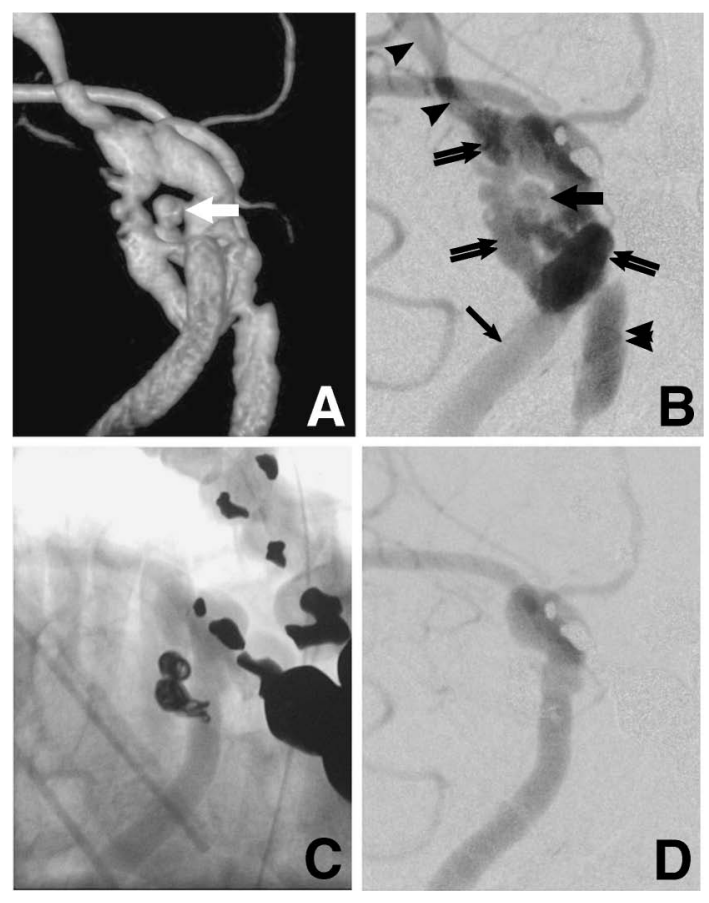

Fig. 2 Angiograms of the right internal carotid artery at the working angle (anteroinferior view). Three-dimensional digital subtraction angiography (3D DSA) (A) and conventional DSA (B) showing the internal carotid artery (arrow), fistula (thick arrows), cavernous sinus (double arrows), superior ophthalmic vein (arrowheads), and inferior petrosal sinus (double arrowhead). Non-subtracted (C) and subtracted (D) images after embolization, showing complete obliteration of the fistula with few coils.

cavernous segment (Fig. 1C). The position of the C-arm was determined as the working angle for intervention, with reference to 3D DSA (Fig. 2A, B). An Excelsior SL-10 microcatheter (Boston Scientific, Natick, Mass., U.S.A.), with a tip shaped in three dimensions using hot steam with reference to the $3 \mathrm{D}$ DSA, was advanced into the cavernous sinus through the fistula. A HyperForm balloon catheter (ev3 Endovascular Inc., Plymouth, M.N., U.S.A.) was placed in the ICA at the orifice of the fistula to prevent 
coil protrusion into the ICA, and to stabilize the microcatheter. The first pouch entered from the ICA was small and had a relatively wide orifice, so was thought to be an aneurysm. We decided to place coils in the second pouch as embolization of only the aneurysm would not be enough to occlude the fistula. Two Guglielmi detachable coils (GDCs) were placed to obliterate the aneurysm using the neck protection technique, after placement of 2 GDCs in the second pouch caused the shunt flow to disappear (Fig. 2C, D). The pulsatile tinnitus disappeared just after the intervention. Ocular symptoms other than visual impairment fully recovered within a week.

\section{Discussion}

Information about the 3D structure of the fistula and the cavernous sinus is very important. Conventional DSA and CT angiography are sometimes useful to detect the fistulous point, but 3D DSA gives more information for devising the therapeutic strategy. ${ }^{3,5)}$ Endoscopic mode is useful to detect the fistulous point if the structure is complicated. The face-cutting technique and the control of window levels on volume-rendered images give useful information to understand the $3 \mathrm{D}$ relationship of the ICA, fistula, and cavernous sinus.

Measurement of the size of the fistula from the 3D DSA images was difficult because it significantly varied depending on the adjustment of the window levels. ${ }^{3)}$ Accurate analyses of large and complicated fistulas may be difficult even with 3D DSA because of insufficient opacification of the lesion with contrast medium. In the present relatively simple case, we were able to devise a therapeutic strategy that included positioning of the C-arm for the working angle, adjustment of the $3 \mathrm{D}$ shape of the tip of the microcatheter, positioning of a balloon catheter for neck protection, identification of the sites to be obliterated with coils, and determination of the size of the coils in reference to volume-rendered images from 3D DSA. Consequently, fewer coils were required for complete obliteration of the fistula and the aneurysm.

Stent-assisted coil embolization is effective for fistulas, particularly for direct high-flow CCFs, caused by a large laceration or rupture of a wide-neck aneurysm. ${ }^{7)}$ Treatment with the covered stent is a promising option for direct CCFs. ${ }^{1)}$ Information such as position, size, and shape of the fistula, which can be provided by 3D DSA, is also important for these techniques.

In conclusion, understanding the $3 \mathrm{D}$ structure of direct CCFs is important for the safe and effective obliteration of fistulas. 3D DSA provides useful information regarding the $3 \mathrm{D}$ anatomical vascular structure around the lesion.

\section{References}

1) Archondakis E, Pero G, Valvassori L, Boccardi E, Scialfa G: Angiographic follow-up of traumatic carotid cavernous fistulas treated with endovascular stent graft placement. AJNR Am J Neuroradiol 28: 342-347, 2007

2) Gupta AK, Purkayastha S, Krishnamoorthy T, Bodhey NK, Kapilamoorthy TR, Kesavadas C, Thomas B: Endovascular treatment of direct carotid cavernous fistulae: a pictorial review. Neuroradiology 48: 831-839, 2006

3) Ishida F, Kojima T, Kawaguchi K, Hoshino T, Murao K, Taki W: Traumatic carotid-cavernous fistula identified by threedimensional digital subtraction angiography-technical note. Neurol Med Chir (Tokyo) 43: 369-373, 2003

4) Jung JY, Kim SH, Kim DJ, Kim DI: Navigation-assisted transsphenoidal deflation of a detachable balloon in the cavernous sinus after embolization of a direct carotid-cavernous fistula. Acta Neurochir (Wien) 149: 207-212, 2007

5) Kwon BJ, Han MH, Kang HS, Chang KH: Endovascular occlusion of direct carotid cavernous fistula with detachable balloons: usefulness of 3D angiography. Neuroradiology 47: 271-281, 2005

6) Linskey ME, Sekhar LN, Hirsch W Jr, Yonas H, Horton JA: Aneurysms of the intracavernous carotid artery: clinical presentation, radiographic features, and pathogenesis. Neurosurgery 26: 71-79, 1990

7) Moron FE, Klucznik RP, Mawad ME, Strother CM: Endovascular treatment of high-flow carotid cavernous fistulas by stent-assisted coil placement. AJNR Am J Neuroradiol 26: 1399-1404, 2005

8) van Rooij WJ, Sluzewski M, Beute GN: Ruptured cavernous sinus aneurysms causing carotid cavernous fistula: incidence, clinical presentation, treatment, and outcome. AJNR Am J Neuroradiol 27: 185-189, 2006

Address reprint requests to: Shinya Kohyama, M.D., Doctor's Office 421, Division of Endovascular Neurosurgery, International Medical Center, Saitama Medical University, 1397-1 Yamane, Hidaka, Saitama 350-1298, Japan. e-mail: skohyama@saitama-med.ac.jp 